\title{
The nature of spacetime singularities
}

\author{
Alan D. Rendall \\ Max-Planck-Institut für Gravitationsphysik \\ Albert-Einstein-Institut \\ Am Mühlenberg 1 \\ 14476 Golm, Germany
}

\begin{abstract}
Present knowledge about the nature of spacetime singularities in the context of classical general relativity is surveyed. The status of the BKL picture of cosmological singularities and its relevance to the cosmic censorship hypothesis are discussed. It is shown how insights on cosmic censorship also arise in connection with the idea of weak null singularities inside black holes. Other topics covered include matter singularities and critical collapse. Remarks are made on possible future directions in research on spacetime singularities.
\end{abstract}

\section{Introduction}

The issue of spacetime singularities arose very early in the history of general relativity and it seems that Einstein himself had an ambiguous relationship to singularities. A useful source of information on the confusion surrounding the subject in the first half century of general relativity is [28. The present article is a survey of the understanding we have of spacetime singularities today.

Before concentrating on general relativity, it is useful to think more generally about the concept of a singularity in a physical theory. In the following the emphasis is on classical field theories although some of the discussion may be of relevance to quantum theory as well. When a physical system is modelled within a classical field theory, solutions of the field equations are considered. If it happens that at some time physically relevant quantities 
become infinite at some point of space then we say that there is a singularity. Since the physical theory ceases to make sense when basic quantities become infinite a singularity is a sign that the theory has been applied beyond its domain of validity. To get a better description a theory of wider applicability should be used. Note that the occurrence of singularities does not say that a theory is bad - it only sets limits on the domain of physical phenomena where it can be applied.

In fact almost any field theory allows solutions with singularities if attention is not restricted to those solutions which are likely to be physically relevant. In this context a useful criterion is provided by the specification of solutions by initial data. This means that we only consider solutions which have the property that there is some time at which they contain no singularities. Then any singularities which occur must be the result of a dynamical evolution. With this motivation, singularities will be discussed in the following in the context of the initial value problem. Only those singularities are considered which develop from regular initial configurations. This has the consequence that linear field theories, such as source-free Maxwell theory, are free of singularities.

In the case of the Einstein equations, the basic equations of general relativity, the notion of singularity becomes more complicated due to the following fact. A solution of the Einstein equations consists not just of the spacetime metric, which describes the gravitational field and the geometry of spacetime, but also the spacetime manifold on which the metric is defined. In the case of a field theory in Newtonian physics or special relativity we can say that a solution becomes singular at certain points of spacetime, where the basic physical quantities are not defined. Each of these points can be called a singularity. On the other hand, a singularity in general relativity cannot be a point of spacetime, since by definition the spacetime structure would not be defined there.

In general relativity the wordline of a free particle is described by a curve in spacetime which is a timelike or null geodesic, for a massive or massless particle respectively. There is also a natural class of time parameters along such a geodesic which, in the timelike case, coincide up to a choice of origin and a rescaling with the proper time in the rest frame of the particle. If the worldline of a particle only exists for a finite time then clearly something has gone seriously wrong. Mathematically this is called geodesic incompleteness. A spacetime which is a solution of the Einstein equations is said to be singular if it is timelike or null geodesically incomplete. Informally we say in this case 
that the spacetime 'contains a singularity' but the definition does not include a description of what a 'singularity' or 'singular point' is. There have been attempts to define ideal points which could be added to spacetime to define a mathematical boundary representing singularities but these have had limited success.

When working practically with solutions of the Einstein equations it is necessary to choose coordinates or other similar auxiliary objects in order to have a concrete description. In general relativity we are free to use any coordinate system and this leads to a problem when considering singularities. Suppose that a metric written in coordinates is such that the components of the metric become infinite as certain values of the coordinates are approached. This could be a sign that there is a spacetime singularity but it could also simply mean that those coordinates break down at some points of a perfectly regular solution. This might be confirmed by transforming to new coordinates where the metric components have a regular extension through the apparent singularities. A way of detecting singularities within a coordinate system is to find that curvature invariants become infinite. These are scalar quantities which measure the curvature of spacetime and if they become infinite this is a sure sign that a region of spacetime cannot be extended. It is still not completely clear what is happening since the singular values of the coordinates might correspond to singular behaviour in the sense of geodesic incompleteness or they might be infinitely far away.

A breakthrough in the understanding of spacetime singularities was the singularity theorem of Penrose [39] which identified general conditions under which a spacetime must be geodesically incomplete. This was then generalized to other situations by Hawking and others. The singularity theorems are proved by contradiction. Their strength is that the hypotheses required are very general and their weakness is that they give very little information about what actually happens dynamically. If the wordline of a particle ceases to exist after finite proper time then it is reasonable to ask for an explanation, why the particle ceased to exist. It is to be expected that some extreme physical conditions play a role. For instance, the matter density or the curvature, representing tidal forces acting on the particle, becomes unboundely large. From this point of view one would like to know that curvature invariants become unbounded along the incomplete timelike or null geodesics. The singularity theorems give no information on this question which is that of the nature of spacetime singularities. The purpose of the following is to explain what is known about this difficult question. 
The hypotheses of the singularity theorems do not include very stringent assumptions about the matter content of spacetime. All that is needed are certain inequalities on the energy-momentum tensor $T_{\alpha \beta}$, the energy conditions 31. Let $V^{\alpha}$ and $W^{\alpha}$ be arbitrary future pointing timelike vectors. The dominant energy condition is that $T_{\alpha \beta} V^{\alpha} W^{\beta} \geq 0$. The strong energy condition is, provided the cosmological constant is zero, equivalent to the condition that $R_{\alpha \beta} V^{\alpha} V^{\beta} \geq 0$ where $R_{\alpha \beta}$ is the Ricci tensor. The weak energy condition is that $T_{\alpha \beta} V^{\alpha} V^{\beta} \geq 0$. The weak energy condition has the simple physical interpretation that the energy density of matter is non-negative in any frame of reference. The vector $V^{\alpha}$ is the four-velocity of an observer at rest in that frame of reference. It is not reasonable to expect that the nature of spacetime singularities can be determined on the basis of energy conditions alone - more detailed assumptions on the matter content are necessary.

It follows from the above discussion that spacetime singularities should be associated with reaching the limits of the physical validity of general relativity. Quantum effects can be expected to come in. If this is so then to go further the theory should be replaced by some kind of theory of quantum gravity. Up to now we have no definitive theory of this kind and so it is not clear how to proceed. The strategy to be discussed in the following is to work entirely within classical general relativity and see what can be discovered. It is to be hoped that this will provide useful input for the future investigation of singularities within a more general context. The existing attempts to study the question of singularities within different approaches to quantum gravity, including the popular idea that quantum gravity should eliminate the singularities of classical general relativity, will not be discussed here. For a discussion of one direction where progress is being made, see the article of M. Bojowald in this volume.

A key question about singularities in general relativity is whether they are a disaster for the theory. If a singularity can be formed and then influence the evolution of spacetime then this means a breakdown of predictability for the theory. For we cannot (at least within the classical theory) predict anything about the influence a singularity will have. A singularity which can causally influence parts of spacetime is called a naked singularity. It is important for the predictive power of general relativity that naked singularities be ruled out. This has been formulated more precisely by Penrose as the cosmic censorship hypothesis [40, [41. In fact there are two variants of this, weak and strong cosmic censorhip. Despite the names neither of these implies the other [54]. Proving the cosmic censorship hypothesis is one of the central 
mathematical problems of general relativity. In fact the task of finding the right formulation of the conjecture is already a delicate one. It is necessary to make a genericity assumption and to restrict the matter fields allowed. More details on this are given in later sections.

One of the most important kinds of singularity in general relativity is the initial cosmological singularity, the big bang. The structure of cosmological singularities is the subject of section 2. Another important kind of singularity is that inside black holes. The recent evolution of ideas about the internal structure of black holes is discussed in section 3. An important complication in the study of singularities resulting from the properties of gravity is that they may be obscured by singularities due to the description of matter. This is the theme of section 4. In section 5 5ingularities are discussed which arise at the threshhold of black hole formation and which are still quite mysterious. Section [6 takes a cautious look at the future of research on spacetime singularities.

\section{Cosmological singularities}

The simplest cosmological models are those which are homogeneous and isotropic, the FLRW (Friedmann-Lemaitre-Robertson-Walker) models with some suitable choice of matter model such as a perfect fluid. In this context it is seen that the energy density blows up at some time in the past. An early question was whether this singularity might be an artefact of the high symmetry. The intuitive idea is that if matter collapses in such a way that particles are aimed so as to all end up at the same place at the same time there will be a singularity. On the other hand if this situation is perturbed so that the particles miss each other the singularity might be removed. On the basis of heuristic arguments, Lifshitz and Khalatnikov [34] suggested that for a generic perturbation of a FLRW model there would be no singularity. We now know this to be incorrect. This work nevertheless led to a very valuable development of ideas in the work of Belinskii, Khalatnikov and Lifshitz [7], [8] which is one of the main sources for our present picture of cosmological singularities.

What was the problem with the original analysis? An ansatz was made for the form of the metric near the singularity and it was investigated how many free functions can be accomodated in a certain formal expansion. It was found that there was one function less than there is in the general so- 
lution of the Einstein equations. It was concluded that the most general solution could not have a singularity. This shows us something about the strengths and weaknesses of heuristic arguments. These are limited by the range of possibilities that have occurred to those producing the heuristics. Nevertheless they may, in expert hands, be the most efficient way of getting nearer to the truth.

It was the singularity theorems, particularly the Hawking singularity theorem, which provided convincing evidence that cosmological singularities do occur for very large classes of initial data. In particular they showed that the presence of a singularity (in the sense of geodesic incompleteness) is a stable property under small perturbations of the FLRW model. Thus a rigorous mathematical theorem led to progress in our understanding of physics. The use of mathematical theorems is very appropriate because the phenomena being discussed are very far from most of our experience of the physical world and so relying on physical intuition alone is dangerous.

The singularity theorems give almost no information on the nature of the singularities. In order to go further it makes sense to attempt to combine rigorous mathematics, heuristic arguments and numerical calculations and this has led to considerable progress.

The picture developed by Belinskii, Khalatnikov and Lifshitz (BKL) has several important elements. These are:

- Near the singularity the evolution of the geometry at different spatial points decouples so that the solutions of the partial differential equations can be approximated by solutions of ordinary differential equations.

- For most types of matter the effect of the matter fields on the dynamics of the geometry becomes negligible near the singularity

- The ordinary differential equations which describe the asymptotics are those which come from a class of spatially homogeneous solutions which constitute the mixmaster model. General solutions of these equations show complicated oscillatory behaviour near the singularity.

The first point is very surprising but a variety of analytical and numerical studies appear to support its validity. The extent to which the above points have been confirmed will now be discussed. 
The mixmaster model is described by ordinary differential equations and so it is a huge simplification compared to the full problem. Nevertheless even ordinary differential equations can be very difficult to analyse. The solutions show complicated behaviour in the approach to the singularity and this is often called chaotic. This description is somewhat problematic since many of the usual concepts for defining chaos are not applicable. This point will not be discussed further here. For many years the oscillations in solutions of the mixmaster model were studied by heuristic and numerical techniques. This led to a consistent picture but turning this picture into mathematical theorems was an elusive goal. Finally this was achieved in the work of Ringström [49] so that the fundamental properties of the mixmaster model are now mathematically established.

With the mixmaster model under control, the next obvious step in confirming the BKL picture would be to show that it serves as a template for the behaviour of general solutions near the singularity. The work of BKL did this on a heuristic level. Attempts to recover their conclusions in numerical calculations culminated in the work of Garfinkle [30]. Previously numerical investigations of the question had been done under various symmetry assumptions. Solutions without symmetry were handled for the first time in [30]. On the analytical side things do not look so good. There is not a single case with both inhomogeneity and mixmaster oscillations which has been analysed rigorously and this represents an outstanding challenge. One possible reason why it is so difficult will be described below.

One of the parts of the BKL picture contains the qualification 'for most types of matter'. There are exceptional types of matter where things are different. A simple example is a massless linear scalar field. It was already shown in [6] that in the presence of a scalar field the BKL analysis leads to different conclusions. It is still true that the dynamics at different spatial points decouples but the evolution is such that important physical quantities are ultimately monotone instead of being oscillatory as the singularity is approached. In this case it has been possible to obtain a mathematical confirmation of the BKL picture. In 2] it was shown that there are solutions of the Einstein equations coupled to a scalar field which depend on the maximal number of free functions and which have the asymptotic behaviour near the singularity predicted by the BKL picture.

As a side remark, note that in many string theory models there is a scalar field, the dilaton, which might kill mixmaster oscillations. Also, a BKL analysis of the vacuum Einstein equations in higher dimensions shows 
that the oscillations of generic solutions vanish when the spacetime dimension is at least eleven 27 and string theory leads to the consideration of models of dimension greater than four. So could mixmaster oscillations be eliminated in low energy string theory? An investigation in [25] shows that they are not. The simplifying effect of the dilaton and the high dimension is prevented by other form fields occurring in string theory. With certain values of the coupling constants in field theories of the type coming up in low energy string theory there is monotone behaviour near the singularity and theorems can be proved [26]. However the work of 25] shows that these do not include the values of the coupling constants coming from the string theories which are now standard.

A feature which makes oscillations so difficult to handle is that they are in general accompanied by large spatial gradients. Consider some physical quantity $f(t, x)$ in the BKL picture in a case without oscillations. Then it is typical that quantities like $\partial_{i} f / f$, where the derivatives are spatial derivatives, remain bounded near the singularity. However it can happen that this is only true for most spatial points and that there are exceptional spatial points where it fails. In a situation of mixmaster type where there are infinitely many oscillations as the singularity is approached the BKL picture predicts that there will be more and more exceptional points without limit as the singularity is approached. It has even been suggested by Belinskii that this shows that the original BKL assumptions are not self-consistent [5]. In any case, it seems that the question, in what sense the BKL picture provides a description of cosmological singularities, is a subtle one.

Large spatial gradients can also occur in solutions where the evolution is monotone near the singularity. It can happen that before the monotone stage is reached there are finitely many oscillations and that these produce a finite number of exceptional points. In the context of Gowdy spacetimes this has been shown rather explicitly. The features with large spatial gradients (spikes) were discovered in numerical work 9] and later captured analytically 48. This allowed the behaviour of the curvature near the singularity to be determined.

An important issue to be investigated concerning cosmological singularities is that of cosmic censorship. In this context it is strong cosmic censorship which is of relevance and a convenient mathematical formulation in terms of the initial value problem has been given by Eardley and Moncrief [37. To any initial data set for the Einstein equations there exists a corresponding maximal Cauchy development. (For background on the initial value prob- 
lem for the Einstein equations see [29].) The condition that a spacetime is uniquely determined by initial data is global hyperbolicity. The maximal Cauchy development is in a well-defined sense the largest globally hyperbolic spacetime with the chosen initial data. It may happen that the maximal Cauchy development can be extended to a larger spacetime, which is then of course no longer globally hyperbolic. The boundary of the initial spacetime in the extension is called the Cauchy horizon. The extended spacetime can no longer be uniquely specified by initial data and this corresponds physically to a breakdown of predictability. A famous example where this happens is the Taub-NUT spacetime [31. This is a highly symmetric solution of the Einstein vacuum equations. The extension which is no longer globally hyperbolic contains closed timelike curves.

How can the existence of the Taub-NUT and similar spacetimes be reconciled with strong cosmic censorship? A way to do this would be to show that this behaviour only occurs for exceptional initial data and that for generic data the maximal globally hyperbolic development is inextendible. This has up to now only been achieved in the simplified context of classes of spacetimes with symmetry. These classes of spacetimes are not generic and so they do not directly say anything about cosmic censorship. However they provide model problems where more can be learned about the conceptual and technical issues which arise in trying to prove cosmic censorship. This kind of restricted cosmic censorship has been shown for many spatially homogeneous spacetimes in [20] and [45] and for plane symmetric solutions of the Einstein equations coupled to a massless scalar field [53]. The most general, and most remarkable, result of this kind up to now is the proof by Ringström [51] of strong cosmic censorship restricted to the class of Gowdy spacetimes. He shows that all the solutions in this class of inhomogeneous vacuum spacetimes with symmetry are geodesically complete in the future [50] and that for generic initial data the Kretschmann scalar $R^{\alpha \beta \gamma \delta} R_{\alpha \beta \gamma \delta}$ tends to infinity uniformly as the singularity is approached. Major difficulties in doing this are the fact that there do exist spacetimes in this class where the maximal Cauchy development is extendible and that spikes lead to great technical complications. Roughly speaking, Ringström shows under a genericity assumption that the most complicated thing that can happen in the approach to the singularity is that there are finitely many spikes of the kind constructed in 48].

Another kind of partial result is to show that an expanding cosmological spacetime is future geodesically complete. This can be interpreted as saying 
that any singularities must lie in the past. There is up to now just one example of this for spacetimes not required to satisfy any symmetry assumptions. This is the work of Andersson and Moncrief [1] where they show that any small but finite vacuum perturbation of the initial data for the Milne model has a maximal Cauchy development which is future geodesically complete.

Already in the class of homogeneous and isotropic spacetimes there are models with an initial singularity which recollapse and have a second singularity in the future. Not much is known about general criteria for recollapse. The closed universe recollapse conjecture [4 says that any spacetime with a certain type of topology (admitting a metric of positive scalar curvature) and satisfying the dominant and strong energy conditions must recollapse. No counterexample is known but the conjecture has only been proved in cases with high symmetry 13, 14.

\section{Black hole singularities}

One of the most famous singular solutions of the Einstein equations is the Schwarzschild solution representing a spherical black hole. There is a singularity inside the black hole where the Kretschmann scalar diverges uniformly. It looks very much like a cosmological singularity. The singularity is not visible to far away observers. The points of spacetime from which no futuredirected causal geodesic can escape to infinity constitute by definition the black hole region and its boundary is the event horizon. The situation in the Schwarzschild solution can be described informally by saying that the singularity is covered by an event horizon. The idea of weak cosmic censorship, a concept which will not be precisely defined here, is that any singularity which arises in gravitational collapse is covered by an event horizon. For more details see [54], 17].

The central question which is to be answered is what properties of the Schwarzschild solution are preserved under perturbations of the initial data. Christodoulou has studied the spherical gravitational collapse of a scalar field in great detail [18. Among his results are the following. There are initial data leading to the formation of naked singularities but for generic initial data this does not happen. The structure of the singularity has been analysed and it shows strong similarities to the Schwarzschild case.

A key concept in the Penrose singularity theorem is that of a trapped surface. It has been shown by Dafermos [22] that some of the results of 
Christodoulou can be extended to much more general spherically symmetric spacetimes under the assumptions that there exists at least one trapped surface and that the matter fields present are well-behaved in a certain sense. They should not form singularities outside the black hole region. This condition on the matter fields was verified for collisionless matter in [24]. The fact that it is satisfied for certain non-linear scalar fields led to valuable insights in the discussion of the formation of naked singularities in a class of models motivated by string theory [32], 23].

When the Schwarzschild solution is generalized to include charge or rotation the picture changes dramatically. In the relevant solutions, the ReissnerNordström and Kerr solutions, the Schwarzschild singularity is replaced by a Cauchy horizon. At one time it was hoped that this was an artefact of high symmetry and that a further perturbation would turn it back into a curvature singularity. There was also a suggested mechanism for this, namely that radiation coming from the outside would undergo an unlimited blue shift as it approached the potential Cauchy horizon. Things turned out to be more complicated, as discovered by Poisson and Israel 42.

The new picture in [42] for a perturbed charged black hole was that the Cauchy horizon, where the metric is smooth, would be replaced by a null hypersurface where, although the metric remains continuous and nondegenerate, the curvature blows up. They called this a weak null singularity. The heuristic work of [42] was followed up by numerical work [33] and was finally turned into rigorous mathematics by Dafermos [21]. Perhaps the greatest significance of this work on charged black holes is its role as a model for rotating black holes. For the more difficult case of rotation much less is known although there is some heuristic analysis [38. At this point it is appropriate to make a comment on heuristic work which follows on from remarks in the last section. For several years it was believed, on the basis of a heuristic analysis in [36, that a positive cosmological constant would stabilize the Reissner-Nordström Cauchy horizon. This turned out, however, to be another case where not all relevant mechanisms had been thought of. In a later heuristic analysis [11] it was pointed out that there is another instability mechanism at work which reverses the conclusion.

The case of weak null singularities draws attention to an ambiguity in the definition of strong cosmic censorship. The formulation uses the concept of extension of a spacetime. To have a precise statement is must be specified how smooth a geometry must be in order to count as an extension. This may seem at first sight like hair splitting but in the case of weak null singularities 
the answer to the question of strong cosmic censorship is quite different depending on whether the extension is required to be merely continuous or continuously differentiable. A related question is whether the extension should be required to satisfy the Einstein equations in some sense.

A question which does not seem to have been investigated is that of the consistency of weak null singularities with the BKL picture. It is typical to study black holes in the context of isolated systems. In reality we expect that black holes form in cosmological models which expand for ever. Do such 'cosmological black holes' show the same features in their interior as asymptotically flat ones? If so then this would indicate the existence of large classes of cosmological models whose singularities do not fit into the BKL picture. (It was never claimed that this picture must apply to all cosmological singularities.) A major difficulty in investigating this issue is that the class of solutions of the Einstein equations of interest does not seem to be consistent with any symmetry assumptions. A related question is that of the relationship between weak cosmic censorship, which is formulated in asymptotically flat spacetimes, and strong cosmic censorship, which makes sense in a cosmological context.

There are important results showing that no black holes form under certain circumstances. In the fundamental work of Christodoulou and Klainerman [19] it was shown that small asymptotically flat data for the Einstein vacuum equations lead to geodesically complete spacetimes. See also [35].

\section{Shells and shocks}

A serious obstacle to determining the structure of spacetime singularites is that many common matter models develop singularities in flat space. This is in particular the case for matter models which are phenomenological rather than coming directly from fundamental physics. These matter models, when coupled to the Einstein equations, must be expected to lead to singularities which have little to do with gravitation which we may call matter singularities. These singularities are just a nuisance when we want to study spacetime singularities as fundamental properties of Einstein gravity.

There has been much study of the Einstein equations coupled to dust. It is not clear that they teach us much. In flat space dust forms shellcrossing singularities where a finite mass of dust particles end up at the same place at the same time. The density blows up there. In curved space 
this leads to naked singularities [55. These occur away from the centre in spherical symmetry. Finite time breakdown of self-gravitating dust can also be observed in cosmological spacetimes [46. This shows the need for restricting the class of matter considered if a correct formulation of cosmic censorship is to be found. In a more realistic perfect fluid the pressure would be expected to eliminate these singularities. On the other hand it is to be expected that shocks form, as is well-known in flat space. The breakdown of smoothness in self-gravitating fluids with pressure was proved in [47. At this point we must once again confront the question of what is a valid extension. In some cases solutions with fluid may be extended beyond the time when the classical solution breaks down [3]. The extended solution is such that the basic fluid variables are bounded but their first derivatives are not. The uniqueness of these solutions in terms of their initial data is not known but uniqueness results have recently been obtained in the flat space case [12].

A matter model which is better behaved than a fluid is collisionless matter described by the Vlasov equation. It forms no singularities in flat space and there are various cases known where self-gravitating collisionless matter can be proved to form no singularities. For instance this is the case for small spherically symmetric asymptotically flat initial data [43. There is no case known where collisionless matter does form a matter singularity. Also in spherical symmetry it never forms a singularity away from the centre so that the analogue of shell-crossing singularities is ruled out [44. In view of the investigations up to now collisionless matter seems to be as well-behaved as vacuum with respect to the formation of singularities.

\section{Critical collapse}

Evidence for a new kind of singularity in gravitational collapse was discovered by Choptuik [16. His original work concerned the spherically symmetric collapse of a massless scalar field but it has been extended in many directions since then. The basic idea is as follows. For small initial data the corresponding solution disperses leaving behind flat space. For very large data a black hole is formed. If a one-parameter family of data is taken interpolating between these two extreme cases what happens to the evolutions for intermediate values of the parameter? It is found that there is a unique parameter value (the critical value) separating the two regimes and that near the critical value the solutions show interesting, more or less universal, behaviour. The 
study of these phenomena is now known under the name of critical collapse.

Most of the work which has been done on critical collapse is numerical. There is a heuristic picture involving dynamical systems which is useful in predicting certain features of the results of numerical calculations. Up to now there are no rigorous results on critical phenomena. It is interesting to note that at least some of the features of critical collapse are not unique to gravity and may be seen in many systems of partial differential equations 10.

The results on critical collapse indicate the occurrence of a class of naked singularities arising from non-generic initial data which are qualitatively different from those discussed above. They represent an additional technical hurdle in any attempt to prove cosmic censorship in general.

\section{Conclusion}

In recent years it has been possible to go beyond the classical results on spacetime singularities contained in the singularity theorems of Penrose and Hawking and close in on the question of the nature of these singularities in various ways. In the case of cosmological singularities a key influence has been exerted by the picture of Belinskii, Khalatnikov and Lifshitz (BKL). In the case of black hole singularities the old idea that they should be similar to cosmological singularities has been replaced by the new paradigm of weak null singularities due to Poisson and Israel. A new kind of singularity has emerged in the work of Choptuik on critical collapse. It remains to be seen whether the Einstein equations have further types of singularities in store for us.

New things can happen if we go beyond the usual framework of the singularity theorems. The cosmological acceleration which is now well-established by astronomical observations corresponds on the theoretical level to a violation of the strong energy condition and suggests that a reworking of the singularity theorems in a more general context is necessary. Exotic types of matter which have been introduced to model accelerated cosmological expansion go even further and violate the dominant energy condition. This can lead to a cosmological model running into a singularity when still expanding [52. This is known as a 'big rip' singularity [15] since physical systems are ripped apart in finite time as the singularity is approached. The study of these matters is still in a state of flux. 
Returning to the more conventional setting where the dominant energy condition is satisfied, we can ask what the future holds for the study of spacetime singularities in classical general relativity. A fundamental fact is that our understanding is still very incomplete. Two developments promise improvements. The first is that the steady increase in computing power and improvement of numerical techniques means that numerical relativity should have big contributions to make. The second is that advances in the theory of hyperbolic partial differential equations are providing the tools needed to make further progress with the mathematical theory of solutions of the Einstein equations. As illustrated by the examples of past successes surveyed in this paper the numerical and mathematical approaches can complement each other very effectively.

\section{References}

[1] Andersson, L. and Moncrief, V. 2004 Future complete vacuum spacetimes. In: Chruściel, P. T. and Friedrich, H. The Einstein equations and the large scale behaviour of gravitational fields. Birkhäuser, Basel.

[2] Andersson, L. and Rendall, A. D. 2001 Quiescent cosmological singularities. Commun. Math. Phys. 218, 479-511.

[3] Barnes, A. P., LeFloch, P. G., Schmidt, B. G. and Stewart, J. M. 2004 The Glimm scheme for perfect fluids on plane-symmetric Gowdy spacetimes. Class. Quantum Grav. 21, 5043-5074.

[4] Barrow, J. D., Galloway, G. J. and Tipler, F. J. 1986 The closed-universe recollapse conjecture. Mon. Not. R. Astron. Soc. 223, 835-844.

[5] Belinskii, V. A. 1992 Turbulence of a gravitational field near a spacetime singularity. JETP Lett. 56, 421-425.

[6] Belinskii, V. A. and Khalatnikov, I. M. 1973 Effect of scalar and vector fields on the nature of cosmological singularities. Sov. Phys. JETP 36, 591-597.

[7] Belinskii, V. A., Khalatnikov, I. M. and Lifshitz, E. M. 1970 Oscillatory approach to a singular point in the relativistic cosmology. Adv. Phys. $19,525-573$. 
[8] Belinskii, V. A., Khalatnikov, I. M. and Lifshitz, E. M. 1982 A general solution of the Einstein equations with a time singularity. Adv. Phys., 31, 639-667.

[9] Berger, B. K. and Moncrief, V. 1994 Numerical investigation of spacetime singularities. Phys. Rev. D48, 4676-4687.

[10] Bizoń, P. and Tabor, Z. 2001 On blowup for Yang-Mills fields. Phys. Rev. D64, 121701.

[11] Brady, P. R., Moss, I. G. and Myers, R. C. 1998 Cosmic censorship: as strong as ever. Phys. Rev. Lett. 80, 3432-3435.

[12] Bressan, A. 2000 Hyperbolic systems of conservation laws. The onedimensional Cauchy problem. Oxford University Press, Oxford.

[13] Burnett, G. 1995 Lifetimes of spherically symmetric closed universes. Phys. Rev. D51, 1621-1631.

[14] Burnett, G. and Rendall, A. D. 1996 Existence of maximal hypersurfaces in some spherically symmetric spacetimes. Class.Quant.Grav. 13, 111124

[15] Caldwell. R. R., Kamionkowski, M. and Weinberg, N. N. 2003 Phantom energy and cosmic doomsday. Phys. Rev. Lett. 91, 071301.

[16] Choptuik, M. W. 1993 Universality and scaling in the gravitational collapse of a scalar field. Phys. Rev. Lett. 70, 9-12.

[17] Christodoulou, D. 1999 On the global initial value problem and the issue of singularities. Class. Quantum Grav. A23-A35,

[18] Christodoulou, D. 1999 The instability of naked singularities in the gravitational collapse of a scalar field. Ann. Math. 149, 183-217.

[19] Christodoulou, D. and Klainerman, S. 1993 The global nonlinear stability of the Minkowski space. Princeton University Press, Princeton.

[20] Chruściel, P. T. and Rendall, A. D. 1995 Strong cosmic censorship in vacuum space-times with compact locally homogeneous Cauchy surfaces. Ann. Phys. 242, 349-385. 
[21] Dafermos, M. 2003 Stability and instability of the Cauchy horizon for the spherically symmetric Einstein-Maxwell-scalar field equations. Ann. Math. 158, 875-928.

[22] Dafermos, M. 2004 Spherically symmetric spacetimes with a trapped surface. Preprint gr-qc/0403032.

[23] Dafermos, M. 2004 On naked singularities and the collapse of selfgravitating Higgs fields. Preprint gr-qc/0403033.

[24] Dafermos, M. and Rendall, A. D. 2004 An extension principle for the Einstein-Vlasov system in spherical symmetry. Preprint gr-qc/0411075.

[25] Damour, T. and Henneaux, M. 2000 Chaos in superstring cosmology. Phys. Rev. Lett. 85, 920-923.

[26] Damour, T., Henneaux, M., Rendall, A. D. and Weaver, M. 2002 Kasnerlike behaviour for subcritical Einstein-matter systems. Ann. H. Poincare 3, 1049-1111.

[27] Demaret, J., Henneaux, M. and Spindel, P. 1985 Nonoscillatory behaviour in vacuum Kaluza-Klein cosmologies. Phys. Lett. 164B, 27-30.

[28] Earman, J. 1995 Bangs, crunches, whimpers and shrieks. Singularities and acausalities in relativistic spacetimes. Oxford University Press, Oxford.

[29] Friedrich, H. and Rendall, A. D. 2000 The Cauchy problem for the Einstein equations. In B. G. Schmidt (ed) Einstein's Field Equations and Their Physical Implications. Lecture Notes in Physics 540. Springer, Berlin.

[30] Garfinkle, D. 2004 Numerical simulations of generic singularities. Phys.Rev.Lett. 93, 161101.

[31] Hawking, S. W. and Ellis, G. F. R. 1973 The large-scale structure of space-time. Cambridge University Press, Cambridge.

[32] Hertog, T., Horowitz, G. T. and Maeda, K. 2004 Generic cosmic censorship violation in anti de Sitter space. Phys. Rev. Lett. 92, 131101. 
[33] Hod, S. and Piran, T. 1998 Mass inflation in dynamical collapse of a charged scalar field. Phys. Rev. Lett. 81, 1554-1557.

[34] Lifshitz, E. M. and Khalatnikov, I. M. 1963 Investigations in relativistic cosmology. Adv. Phys. 12, 185-249.

[35] Lindblad, H. and Rodnianski, I. 2004 The global stability of Minkowski space-time in harmonic gauge. Preprint math.AP/0411109.

[36] Mellor, F., Moss, I. G. 1990 Stability of black holes in de Sitter space. Phys. Rev. D41, 403-409.

[37] Moncrief, V. and Eardley, D. M. 1981 The global existence problem and cosmic censorship in general relativity. Gen. Rel.Grav. 13, 887-892.

[38] Ori, A. 1999 Oscillatory null singularity inside realistic spinning black holes. Phys. Rev. Lett. 83, 5423-5426.

[39] Penrose, R. 1965 Gravitational collapse and spacetime singularities. Phys. Rev. Lett. 14, 57-59.

[40] Penrose, R. 1969 Gravitational collapse: the role of general relativity. Riv. del Nuovo Cimento 1, 252-276.

[41] Penrose, R. 1979 Singularities and time asymmetry. In: Hawking, S. W. and Israel, W. General relativity: an Einstein centenary survey. Cambridge University Press, Cambridge.

[42] Poisson, E. and Israel, W. 1990 Internal structure of black holes. Phys. Rev. D41, 1796-1809.

[43] Rein, G. and Rendall, A. D. 1992 Global existence of solutions of the spherically symmetric Vlasov-Einstein system with small initial data. Commun. Math. Phys. 150, 561-583.

[44] Rein, G., Rendall, A. D. and Schaeffer, J. 1995 A regularity theorem for solutions of the spherically symmetric Vlasov-Einstein system. Commun. Math. Phys. 168, 467-478.

[45] Rendall, A. D. 1995 Global properties of locally spatially homogeneous cosmological models with matter. Math. Proc. Camb. Phil. Soc. 118, 511-526. 
[46] Rendall, A. D. 1997 Existence and non-existence results for global constant mean curvature foliations. Nonlinear Analysis, Theory, Methods and Applications 30, 3589-3598.

[47] Rendall, A. D. and Ståhl, F. 2005 Shock waves in plane symmetric spacetimes. Unpublished.

[48] Rendall, A. D. and Weaver, M. 2001 Manufacture of Gowdy spacetimes with spikes. Class. Quantum Grav. 18, 2959-2975.

[49] Ringström, H. 2001 The Bianchi IX attractor. Ann. H. Poincaré 2, 405500 .

[50] Ringström, H. 2004 On a wave map equation arising in general relativity. Commun. Pure Appl. Math. 57, 657-703.

[51] Ringström, H. 2004 Strong cosmic censorship in $T^{3}$ Gowdy spacetimes. Preprint.

[52] Starobinsky, A. A. 2000 Future and origin of our universe: modern view. Grav. Cosmol. 6, 157-163.

[53] Tegankong, D. 2005 Global existence and future asymptotic behaviour for solutions of the Einstein-Vlasov-scalar field system with surface symmetry. Preprint gr-qc/0501062.

[54] Wald, R. M. 1997 Gravitational collapse and cosmic censorship. Preprint gr-qc/9710068.

[55] Yodzis, P., Seifert, H.-J. and Müller zum Hagen, H. 1973 On the occurrence of naked singularities in general relativity. Commun. Math. Phys. $34,135-148$. 